\title{
Unusual complication of multiple splenic abscesses arising from a feeding jejunostomy tube subsequent to total gastrectomy: A case report and literature review
}

\author{
SHUCHANG AN ${ }^{1}$, BING LI $^{1}$, RONG CUI ${ }^{1}$, FENG YAN $^{1}$, GUOSHAN YANG $^{2}$, \\ LI ZHAO $^{2}$, ZHENYA ZHANG ${ }^{2}$ and RUIQIN WANG ${ }^{1}$
}

Departments of ${ }^{1}$ Respiratory Medicine and ${ }^{2}$ General Surgery, First Hospital of Tsinghua University, Beijing 100016, P.R. China

Received June 25, 2014; Accepted February 11, 2015

DOI: $10.3892 / 01.2015 .3007$

\begin{abstract}
Splenic abscess is a rare clinical entity. The present study reports a case of a patient that suffered from splenic abscess secondary to septicemia resulting from Klebsiella pneumoniae infection following the removal of the feeding jejunostomy tube that was utilized subsequent to the patient undergoing total gastrectomy as part of the treatment regimen for gastric adenocarcinoma. The early clinical presentation was nonspecific and multiple splenic abscesses were subsequently identified. To reduce the risks of an additional surgical procedure in this particular patient, laparoscopic assisted splenotomy and catheter drainage were performed. Due to the severe complications that occurred in the present patient, no adjuvant chemotherapy was administered. Therefore, the unusual complication of splenic abscess subsequent to total gastrectomy should be noted, and the routine feeding jejunostomy tube placement at the time of total gastrectomy should be discussed and re-assessed.
\end{abstract}

\section{Introduction}

D2 radical gastrectomy is currently considered to be the standard procedure for the treatment of gastric cancer. Due to the more extensive anatomic separation and resection compared with conventional gastrectomy surgery, the incidence of intraoperative-associated injury and post-operative complications is significantly increased $(1,2)$. The overall morbidity and mortality rates of post-operative complications have been reported as $38.4 \%$ and $2.7 \%$, respectively (1). Subsequent to gastrectomy, patients are vulnerable to malnutrition from reduced oral intake, which contributes to the increased risk of post-operative complications and morbidity (3). Therefore,

Correspondence to: Dr Shuchang An, Department of Respiratory Medicine, First Hospital of Tsinghua University, 6 Jiuxianqiao 1st Road, Beijing 100016, P.R. China

E-mail: anshuchang@sohu.com

Key words: splenic abscess, feeding jejunostomy tube, total gastrectomy the insertion of a feeding jejunostomy tube (J-tube) at the time of gastroesophageal resection is recommended to optimize post-operative nutritional status or facilitate adjuvant therapy following surgery $(4,5)$. The insertion procedure is routinely performed. However, the benefit of feeding tube placement and post-operative enteral nutrition continue to be debated due to the possible complications caused by infection that can result from the procedure $(6,7)$. The present study describes a case in which the patient developed the unusual complication of multiple splenic abscesses secondary to the removal of the J-tube, subsequent to total gastrectomy. Therefore the routine management of J-tube placement at the time of total gastrectomy may be questionable. To the best of our knowledge, this is the first reported case of septicemia combined with splenic abscess subsequent to total gastrectomy. The patient provided written informed consent.

\section{Case report}

A 75-year-old male presented to the First Hospital of Tsinghua University (Beijing, China) with chills and hyperpyrexia, and was subsequently referred to the Department of Respiratory Medicine. The patient had undergone a standard D2 total gastrectomy and intra-operative insertion of a feeding J-tube due to gastric adenocarcinoma at Peking University People's Hospital (Beijing, China) six weeks prior. The patient had suffered from mild pneumonia on post-operative day three and had recovered quickly subsequent to antibiotic therapy. The patient was discharged on post-operative day 10 and initiated full oral feeding despite the presence of the J-tube. The postdischarge course was uneventful. The J-tube was removed on post-operative day 40 and then the patient presented a sudden hyperpyrexia accompanied by chills and a mild cough on the following day. The patient possessed a background of coronary heart disease and unstable angina pectoris of a 40-year duration, type 2 diabetes mellitus of a 20-year duration, and had experienced a mild cerebral infarction 10 years previously. On the day of admission, a physical examination revealed a body temperature of $39.3^{\circ} \mathrm{C}$, blood pressure of $160 / 80 \mathrm{mmHg}$ and heart rate of 100 beats/min. The abdominal jejunostomy fistula appeared to be clean and scabbed. The results of other examinations appeared to be normal, with the exception of 


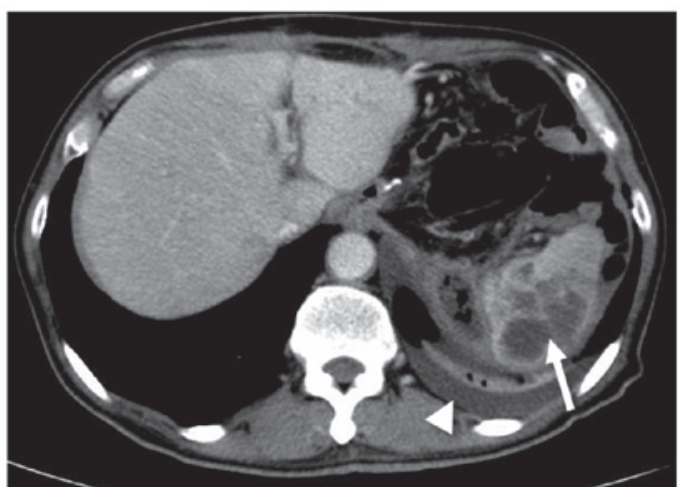

Figure 1. Representative image of an enhanced abdominal computed tomography scan. Multiple non-homogeneous low-density lesions (arrow) with ring enhancement were revealed within the spleen, and reactive left pleural effusion (triangle) was also identified.



Figure 2. Non-enhanced computed tomography scan performed in April 2014 revealed two small low-density lesions (arrow) within the spleen.

being underweight and demonstrating a body mass index of 17.3 (normal range, 18.5-23.9). The laboratory data revealed a white blood cell (WBC) count of $7.3 \times 10^{9} / 1$ (normal range, 4.0-10.0 $\times 10^{9} / 1$ ), containing $79.6 \%$ neutrophils (normal range, $53.0-75.0 \%$ ), a serum sodium level of $118 \mathrm{mmol} / 1$ (normal range, $133-146 \mathrm{mmol} / \mathrm{l})$, a serum potassium level of $4.2 \mathrm{mmol} / \mathrm{l}$ (normal range, 3.3-5.1 mmol/1), a serum albumin level of $27.3 \mathrm{~g} / \mathrm{l}$ (normal range, 35.0-52.0 g/l) and a blood glucose level of $16.5 \mathrm{mmol} / \mathrm{l}$ (normal range, 3.9-6.1 mmol/l). The patient was treated with once-daily intravenous injection of $500 \mathrm{mg}$ levofloxacin. The following day, the patient developed rigor and a body temperature of $39.8^{\circ} \mathrm{C}$, resulting in treatment with intravenous injections of $1 \mathrm{~g}$ imipenem/cilastatin sodium three times daily. After two days, the body temperature returned to normal and Klebsiella pneumoniae was detected in blood culture. The patient then chose to be transferred to the Third Central Hospital of Baoding City (Baoding, China) and was only treated with the imipenem/cilastatin sodium regimen for an additional five days.

On the seventh day after termination of the treatment regimen, the patient presented again with hyperpyrexia, possessing a body temperature of $39.5^{\circ} \mathrm{C}$. Subsequent to four days of treatment with intravenous injections of $4.5 \mathrm{~g}$ piperacillin/tazobactam twice daily at the local hospital, the condition of the patient demonstrated no improvement. Therefore, the patient was again admitted to the Department of Respiratory Medicine of the First Hospital of Tsinghua University. The second set of laboratory data revealed a WBC count of $9.0 \times 10^{9} / 1$ (normal range, 4.0-10.0 $\times 10^{9} / 1$ ), containing $67.7 \%$ neutrophils (normal range, 53.0-75.0\%), a serum albumin level of $26.9 \mathrm{~g} / \mathrm{l}$ (normal range, 35.0-52.0 g/l) and a procalcitonin level of $3.6 \mathrm{ng} / \mathrm{ml}$ (normal range, $0-0.1 \mathrm{ng} / \mathrm{ml}$ ). Based on these findings, the patient was diagnosed with septicemia and insufficient antibiotic treatment. The imipenem/cilastatin regimen was initiated again. The following day, the body temperature returned to normal. Nevertheless, on the fourth day after the second admission, the patient experienced rigor again. An abdominal computed tomography (CT) scan was then performed and multiple non-homogeneous low-density lesions with ring enhancement located within the spleen and left pleural effusion were revealed (Fig. 1). The patient was diagnosed with multiple splenic abscesses and was transferred to the Department of General Surgery immediately. On the following day, an ultrasonography (US)-guided percutaneous aspiration was performed and $40 \mathrm{ml}$ of pus was obtained. A percutaneous drainage catheter was then inserted. The pus culture also indicated the presence of $K$. pneumonia. On the sixth day after transference, a laparoscopic-assisted splenotomy was performed and another catheter was inserted due to inadequate drainage. A combination of $4.5 \mathrm{~g}$ piperacillin and tazobactam was also administered twice daily for three days. The patient's body temperature rapidly returned to normal. The catheter was removed one week later. The abscess cavity was revealed to have decreased in size by US and the patient was discharged on post-splenotomy day 14. The condition of the patient was stable at the eight-month follow-up, and only two small low-density lesions were revealed within the spleen by a non-enhanced CT scan (Fig. 2). Due to this severe complication, no adjuvant chemotherapy was administered.

\section{Discussion}

Prior to 1970, the diagnosis of splenic abscess was one of exclusion, and a delay prior to treatment was frequent (8). Recently, the diagnosis of splenic abscesses appears to be increasing in frequency, and one contributing factor may be the increasing number of patients who are immunocompromised and or suffer from cancer (9-11). The predisposing conditions for splenic abscesses include metastatic hematogenous infection, trauma, infection in an adjacent site, and immunodeficiency $(12,13)$, particularly in immunocompromised patients with acquired immune deficiency syndrome (10), those with diabetes or patients using immunosuppressive agents $(11,14)$. With the development of medical technology, novel predisposing factors have emerged for splenic abscesses. Splenic infarction and the subsequent splenic abscess can be induced by laparoscopic Nissen fundoplication (15) or laparoscopic sleeve gastrectomy (16). Splenic abscesses can also be encountered when endoscopic injection of Histoacryl is established as an effective therapy for bleeding gastric varices (17). The most probable cause of the formation of these post-operative abscesses may be the insufficient perfusion and ischemia of the pole of the spleen due to the ligation of the short gastric vessels (SGVs) during the procedures $(15,16)$. 
Common clinical manifestations of splenic abscesses exhibited by patients include chills, fever, upper left-side abdominal pain, and leukocytosis $(9,11,13,18)$. Other symptoms include nausea and vomiting or a left pleural effusion $(9,13,18)$. However, due to the nonspecific nature of the symptoms and signs developed from splenic abscess, the diagnosis of abscess has frequently been delayed in the past and the incidence of morbidity and mortality was occasionally high (9). Notably, the mortality rate in undiagnosed or untreated cases has been reported as $100 \%(12,14)$. From the culture of the abscess, a wide range of aerobic microbes has been obtained, including Staphylococcus, Escherichia coli, and Salmonella. Other reported microbes include fungi and anaerobic organisms (12). However, the gram-negative bacilli have become the predominant microbes associated with splenic abscess due to geographical variations and population differences $(9,11)$. The modern imaging modalities are sensitive tools for the diagnosis of splenic abscess. The sensitivity of CT for the diagnosis of splenic abscess can reach $96 \%$, and that of US can reach $82 \%$ (12).

Splenectomy has long been considered the standard treatment for bacterial splenic abscess (9,12-14). Chang et al (19) suggested that early surgical intervention should be encouraged in patients with risk factors such as multiple splenic abscesses, gram-negative bacillus infection and high acute physiology and chronic health evaluation II scores. However, the strategy for surgery remains debatable in certain patients due to the consideration of complications (18). Although laparoscopic splenectomy has been revealed to be a feasible and safe procedure (20), laparoscopic-assisted splenotomy may be preferred for splenic abscess patients who are at risk of requiring technically demanding procedures, particularly the post-operative occurrences of splenic abscesses with unavoidable fibrous adhesions, congestive splenomegaly, and perisplenitis.

In the present study, the main septicemia symptoms, including rigor, fever and positive culture in blood, were immediately present subsequent to the removal of the J-tube on post-gastrectomy day 40 . It was hypothesized that there is a timedependent association between the removal of the J-tube and the onset of symptoms. It was hypothesized that the infection began in the sinus tract, perhaps as a result of a lack of healing due to the presence of diabetes and malnutrition, and enteric bacterium entered in the vessel through a tiny breakage. Due to the presence of septicemia and inappropriate antibiotic therapy, the enteric bacterium spread to the spleen and produced the metastatic abscess. In addition, the collateral circulation in the spleen may be damaged due to the division of the SGVs during the total gastrectomy, which was proposed to promote the complication in the present patient. During the progression of the disease, the diagnosis of splenic abscess was neglected partly due to the absence of the classic triad of fever, leukocytosis and left-upper quadrant abdominal pain. In consideration of possible postoperative dense fibrous adhesions and the intense inflammatory process around the congestive spleen, the laparoscopic assisted splenotomy and catheter drainage were performed and splenectomy was avoided. Patel et al (7) suggested that the routine use of J-tubes subsequent to subtotal gastrectomy was not justified due to the increased post-operative complications. The present study concluded that the routine placement of the J-tube at the time of resection for total gastrectomy requires reassessment due to the serious complications that arise in certain patients.
In conclusion, the routine feeding jejunostomy at the time of total gastrectomy may be of no clinical benefit or inadvisable for certain patients. The unusual complication of splenic abscess subsequent to gastrectomy should be considered in patients in spite of the absence of classic manifestations. To reduce the risk of complications associated with a repeat surgical procedure on a post-operative patient, laparoscopic assisted splenotomy may remain a selective indication in certain patients with multiple abscesses.

\section{References}

1. Gil-Rendo A, Hernández-Lizoain JL, Martínez-Regueira F, et al: Risk factors related to operative morbidity in patients undergoing gastrectomy for gastric cancer. Clin Transl Oncol 8: 354-361, 2006.

2. Songun I, Putter H, Kranenbarg EM, Sasako M and van de Velde CJ: Surgical treatment of gastric cancer: 15-year follow-up results of the randomized nationwide Dutch D1D2 trial. Lancet Oncol 11: 439-449, 2010.

3. No authors listed: Perioperative total parenteral nutrition in surgical patients. The Veterans Affairs Total Parenteral Nutrition Cooperative Study Group. N Engl J Med 325: 525-532, 1991.

4. Chin KF, Townsend S, Wong W and Miller GV: A prospective cohort study of feeding needle catheter jejunostomy in an upper gastrointestinal surgical unit. Clin Nutr 23: 691-696, 2004.

5. Llaguna OH, Kim HJ, Deal AM, Calvo BF, Stitzenberg KB and Meyers MO: Utilization and morbidity associated with placement of a feeding jejunostomy at the time of gastroesophageal resection. J Gastrointest Surg 15: 1663-1669, 2011.

6. Srinathan SK, Hamin T, Walter S, Tan AL, Unruh HW and Guyatt G: Jejunostomy tube feeding in patients undergoing esophagectomy. Can J Surg 56: 409-414, 2013.

7. Patel SH, Kooby DA, Staley CA III and Maithel SK: An assessment of feeding jejunostomy tube placement at the time of resection for gastric adenocarcinoma. J Surg Oncol 107: 728-734, 2013.

8. Sarr MG and Zuidema GD: Splenic abscess - presentation, diagnosis, and treatment. Surgery 92: 480-485, 1982.

9. Tung CC, Chen FC and Lo CJ: Splenic abscess: an easily overlooked disease? Am Surg 72: 322-325, 2006.

10. Llenas-García J, Fernández-Ruiz M, Caurcel L, Enguita-Valls A, Vila-Santos J and Guerra-Vales JM: Splenic abscess: a review of 22 cases in a single institution. Eur J Intern Med 20: 537-539, 2009.

11. Song FL, Lu LX, Li CX, Yu XZ and Li Y: A retrospective analysis of 19 splenic abscess patients. Zhonghua Nei Ke Za Zhi 52: 313-317, 2013 (In Chinese).

12. Nelken N, Ignatius J, Skinner M and Christensen N: Changing clinical spectrum of splenic abscess. A multicenter study and review of the literature. Am J Surg 154: 27-34, 1987.

13. Green BT: Splenic abscess: report of six cases and review of the literature. Am Surg 67: 80-85, 2001.

14. Ooi LL and Leong SS: Splenic abscesses from 1987 to 1995. Am J Surg 174: 87-93, 1997.

15. Martínez DG, Sánchez AW and García AP: Splenic abscess after laparoscopic Nissen fundoplication: a consequence of short gastric vessel division. Surg Laparosc Endosc Percutan Tech 18: 82-85, 2008.

16. Stamou KM, Menenakos E, Gomatos IP, Panousopoulos SG, Smparounis S, Leandros E and Zografos G: Clinical implications of sleeve gastrectomy as a source of spleen infarction or ischemia. Obes Surg 21: 1490-1493, 2011.

17. Chang CJ, Su CW and Hou MC: Abdominal pain after endoscopic hemostasis of gastric tumor bleeding. Splenic infarction with abscess formation. Gastroenterology 137: e7-e8, 2009.

18. Lee WS, Choi ST and Kim KK: Splenic abscess: a single institution study and review of the literature. Yonsei Med J 52: 288-292, 2011.

19. Chang KC, Chuah SK, Changchien CS, et al: Clinical characteristics and prognostic factors of splenic abscess: a review of 67 cases in a single medical center of Taiwan. World J Gastroenterol 12: 460-464, 2006.

20. Feldman LS: Laparoscopic splenectomy: standardized approach. World J Surg 35: 1487-1495, 2011. 